\title{
Reducing the Unintended Dislodgement of Gastrostomy Tubes in a Long-Term Acute Care Hospital: A QA/QI Pilot Study
}

\author{
Jamil Shah ${ }^{\mathrm{a}, \mathrm{c}}$, Abul Shahidullah ${ }^{\mathrm{b}}$, Stanlee Richards ${ }^{\mathrm{b}}$
}

\begin{abstract}
Background: Since their introduction in 1980, gastrostomy tubes have become effective means of providing both short-term and longterm enteral access and nutritional support. These feeding tubes are ubiquitous in many health care facilities that care for the elderly, but carry high rates of unintended dislodgement - a complication that, if not detected promptly, is associated with substantial morbidity and health care costs. This study determined the dislodgment rate of gastrostomy tubes at 90 days in a cohort of 221 patients and tested the hypothesis that the implementation of a concise protocol to care for patients' gastrostomy tubes would reduce these unintended dislodgements.
\end{abstract}

Methods: The dislodgment rate of gastrostomy tubes at 90 days in a cohort of 221 patients was determined. In addition, a randomized controlled trial was conducted in a long-term acute care hospital in which patients were alternately allocated to either of two geographically separate units: 1) a selected unit where a concise protocol to care for patients' gastrostomy tubes was implemented, and 2) a separate unit where standard care was provided. Enrollment included patients diagnosed with dysphagia - who were receiving mechanical ventilatory support for chronic respiratory failure - who were being administered feedings, fluids and medications via a balloon gastrostomy tube. The primary endpoint was the number of unintended dislodgements of gastrostomy tubes during a 90-day study period.

Results: In a cohort of 221 patients with balloon gastrostomy tubes placed that was observed for a period of 90 days, 64 (29.0\%) had unintended gastrostomy tube dislodgement $(\mathrm{P}<0.028)$. A total of 34 patients were enrolled in the randomized controlled trial with 17 in the treatment group and 17 in the control group. All subjects were followed for a maximum of 90 days. During the study period, there was

Manuscript submitted September 2, 2018, accepted September 24, 2018

aDivision of Gastroenterology and Hepatology, Department of Medicine, Rutgers New Jersey Medical School, University Hospital, 185 South Orange Avenue, Medical Science Building, Room H-538, Newark, NJ 07103, USA

${ }^{b}$ Department of Medicine, Henry J. Carter Specialty Hospital and Nursing Facility, 1752 Park Avenue, New York, NY 10035, USA

${ }^{\mathrm{c} C}$ orresponding Author: Jamil Shah, 899 Woodmere Drive, Valley Stream, NY 11581, USA.Email: jshahid00@gmail.com

doi: https://doi.org/10.14740/gr1084w one episode of unintended gastrostomy tube dislodgement $(5.9 \%)$ in the treatment group, compared with six episodes $(35.3 \%)$ in the control group $(\mathrm{P}<0.047)$ and the previous cohort of 221 patients $(\mathrm{P}<$ $0.028)$.

Conclusion: This study showed a significant reduction in dislodgements after implementation of a protocol that is an innovative, straightforward and economical solution to the problem of the unintended dislodgement of gastrostomy tubes.

Keywords: Gastrostomy tubes; PEG tubes; Unintended dislodgement; Complications of gastrostomy tubes; Complications of PEG tubes

\section{Introduction}

Adequate nutrition and the delivery of essential nutrients, minerals and electrolytes are imperative for growth, wound healing, tissue repair and a source of energy to perform daily tasks. Unnecessary interruptions of feeding are thus not acceptable in ill patients, particularly in those who are unable to have oral nutrition. Such interruptions are a key contributor to patients not attaining their prescribed nutrition goals [1]. The gastrostomy tube is generally regarded as "one of the most useful and established" techniques available of delivering enteral nutrition to these individuals, many of whom reside in acute rehabilitation facilities, nursing homes, skilled nursing facilities, assisted living centers and extended care centers [2].

The procedure of gastrostomy tube placement is performed most commonly through the endoscopic approach, known as percutaneous endoscopic gastrostomy (PEG). It usually takes only $15-20 \mathrm{~min}$. Moreover, the procedure can be performed under sedation instead of general anesthesia, making it ideal, not just for clinically stable patients, but also for critically ill patients [3]. The gastrostomy tube can also be inserted through the surgical approach as well as by interventional radiology (IR).

The routine use of gastrostomy tubes appears simple. However, experience is required in order to avoid complications as well as the involvement of a multidisciplinary health care team to provide optimal gastrostomy tube care [4]. Health care providers should be able to recognize the risk factors associated with complications. Nurses play an integral role through 
administering feedings, performing skin care and recognizing complications at an early stage in order to decrease morbidity and mortality.

A typical gastrostomy tube provides direct access to the gastric lumen and delivers nutrition and medications through a long, flexible feeding tube. The type of gastrostomy tube used in this study has an internal balloon inflated with water. The underlying principle is that, over time, the balloon gradually loses water or can rupture. When the balloon volume is no longer sufficient, the gastrostomy tube slides out from the tract spontaneously. This is a frequent complication from which significant morbidity and mortality can occur [5-7]. However, gastrostomy tubes can usually be replaced at the bedside rather easily by experienced medical staff.

Among the many complications related to gastrostomy tubes is that of unintended dislodgement, which can occur at any time and result in further complications. For example, the internal balloon can collapse and slide out through the gastrostomy tract but remain in the subcutaneous tissue, causing buried bumper syndrome [8]. This is an unusual late complication due to migration of the inner balloon through or into the abdominal wall $[9,10]$. Feeding may continue until the inner end of the gastrostomy tract completely closes, at which time feeding is obstructed. If the bumper remains in the subcutaneous tissue for several days, it can lead to infection and ultimately an abdominal wall abscess that may require surgery. In fact, failure to diagnose buried bumper syndrome can lead to major complications, including gastrointestinal bleeding, perforation of the stomach, peritonitis and death [11, 12]. However, if this condition is identified at an early stage of partial dislodgement, the gastrostomy tube can be repositioned easily into the stomach.

From time to time, the gastrostomy tube slides out completely from the tract and is discovered by the side of the patient. If it does not occupy the tract, the stoma closes - most of the time, within $1-2 \mathrm{~h}$. When this occurs, a new gastrostomy tube cannot be inserted into the stomach via the gastrostomy.

Furthermore, when complications occur, the gastrostomy tube cannot be used for feeding. These patients require urgent nasogastric tube insertion for administering feedings and medications. The patient requires another invasive procedure later on to reinsert a gastrostomy tube. Moreover, these complications can occur at any time, often placing an additional burden on the skeleton staff on duty.

The complication of unintended dislodgement has been underestimated and underappreciated. In order to ensure uninterrupted feeding, gastrostomy tube care should be emphasized. The aim of this study was to investigate the hypothesis that the implementation of a concise protocol to care for patients' gastrostomy tubes would reduce these unintended dislodgements, and, in doing so, improve the quality of life for individual patients.

\section{Materials and Methods}

\section{Study design, study population and selection criteria}

As part of a quality assurance (QA)/quality improvement (QI)

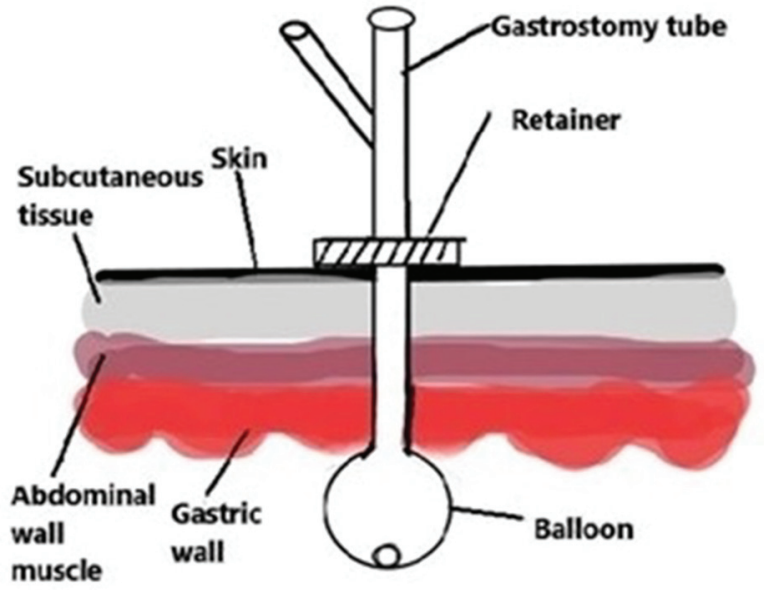

Figure 1. Balloon type gastrostomy tube with the balloon on the inner side of the gastrostomy tract and the retainer on the external side. Reprinted with permission from: Shah JM, Shahidullah AB. Gastric Ulcer from the Pressure of a Gastrostomy Tube: A Rare Cause of Upper Gastrointestinal Bleeding. Cureus. 2018 Jun 11;10(6):e2783. doi: 10.7759/ cureus. 2783.

pilot study, a randomized controlled trial was conducted in a long-term acute care hospital in New York City. The Institutional Review Board of the hospital approved the study. Patient care was managed by a health care team that included a gastroenterologist, medical attending physicians and nurses. Enrollment included patients diagnosed with dysphagia - who were receiving mechanical ventilatory support for chronic respiratory failure - who were being administered feedings, fluids, and medications via a balloon gastrostomy tube (Fig. 1). Patients with gastrostomy tubes of the type with an internal bumper made up a minority of the patients with gastrostomy tubes within the hospital and were not included in the study.

At the beginning of the randomized controlled trial, patients were alternately allocated to either of two geographically separate units: 1) a selected unit where a concise protocol to care for patients' gastrostomy tubes was implemented, and 2) a separate unit where standard care was provided. The protocol was as follows:

Each time a gastrostomy tube was used, its position as well as its mobility in the gastrostomy tract would be checked using markings on the tube (usually $3-6 \mathrm{~cm}$ markings at the opening, depending upon the estimated thickness of the abdominal wall). If there were less than $3 \mathrm{~cm}$ markings at the opening, a physician would examine the gastrostomy tube.

Each balloon gastrostomy tube would have the volume of water in the balloon measured every week. If it was below the manufacturers' recommendations (usually $15 \mathrm{~mL}$ ), water would be added. Also, these gastrostomy tubes would be replaced routinely every 3 months. Thus, unintentional dislodgement could be minimized by checking every 7 days to ensure that the balloon volume was adequate.

All unintended balloon gastrostomy tube dislodgements in both units were recorded during a 90-day study period. Patients were followed through the entirety of the 90-day study period unless they were discharged early from the hospital, had 
Table 1. Age, Gender and Days Observed in Treatment Versus Control Groups

\begin{tabular}{llll} 
& Treatment $(\mathbf{n}=\mathbf{1 7})$ & Control $(\mathbf{n}=\mathbf{1 7})$ & P \\
\hline Patient age, mean (SD) & $71.76(13.71)$ & $70.12(18.98)$ & 0.774 \\
Days observed, mean (SD) & $63.82(26.73)$ & $66.59(29.79)$ & 0.778 \\
Male gender, N (\%) & $7(41.2)$ & $10(58.8)$ & 0.303 \\
\hline
\end{tabular}

Table 2. Unintended Gastrostomy Tube Dislodgements in Treatment Versus Control and Historical Groups

\begin{tabular}{lllll}
\hline \multirow{2}{*}{ Group } & \multicolumn{4}{c}{ Unintended gastrostomy tube dislodgements } \\
\cline { 2 - 5 } & No & Yes & Total & $17(100.0)$ \\
\hline Treatment, N (\%) & $16(94.1)$ & $1(5.9)$ & $17(100.0)$ & 0.047 \\
Control, N (\%) & $11(64.7)$ & $6(35.3)$ & $221(100.0)$ & 0.028 \\
\hline Historical, N (\%) & $157(71.0)$ & $64(29.0)$ & & \\
\hline
\end{tabular}

the gastrostomy tube removed intentionally, or patient mortality occurred.

\section{Statistical analysis}

Treatment and control groups were compared to rule out differences in demographic characteristics and days observed using independent samples $t$-tests and Pearson chi-square analyses. The incidence of unintended gastrostomy tube dislodgements in the treatment group was compared to the incidence in the concurrent control group and in the historical group using onetailed Fisher's exact tests. Additional $t$-tests and chi-square analyses were performed to confirm that the dislodgements were not significantly associated with age, gender or the number of days observed.

\section{Results}

This study determined the dislodgment rate of gastrostomy tubes at 90 days in a cohort of 221 patients and tested the hypothesis that the implementation of a concise protocol to care for patients' gastrostomy tubes would reduce these unintended dislodgements.

In order to do this, a total of 34 patients (17 in the treatment group and 17 in the control group) were enrolled in the study. Patient age ranged from 36 to 100 years, with an average age of 70.9 years ( \pm 16.3 years). An equal number of males and females were enrolled. Treatment and control groups were similar in terms of age and gender (Table 1). Patients who were not followed for the entire 90-day study period could not complete the study because of early discharge from the hospital, recovery of functional swallow leading to intentional removal of the gastrostomy tube, or death. Patients were observed for an average of 65.2 days ( \pm 27.9 days). Treatment and control groups were similar in the number of days observed.

During the trial period, there was only one episode of unintended gastrostomy tube dislodgement $(5.9 \%)$ in the treatment group, compared with six episodes $(35.3 \%)$ in the control group, which constituted a significant difference. Prior to the study, 221 patients with balloon gastrostomy tubes placed were observed for a period of 90 days, and 64 unintended gastrostomy tube dislodgements $(29.0 \%)$ occurred. When compared with the treatment group, the incidence of dislodgements was significantly greater in the historical group (Table 2).

A final set of comparisons confirmed that the incidence of unintended gastrostomy tube dislodgements across both the treatment and control groups was not significantly associated with age, gender or the number of days observed (Table 3).

\section{Discussion}

Gastrostomy tubes are beneficial, cost-effective feeding tubes that can be cared for easily. They are frequently used in longterm care settings. Unfortunately, their use is associated with a range of complications, such as unintended dislodgement. Failure to identify and address these complications early and

Table 3. Age, Gender and Days Observed in All Patients Experiencing Unintended Gastrostomy Tube Dislodgements Versus Those With No Dislodgements

\begin{tabular}{llll} 
& \multicolumn{2}{c}{ Unintended Gastrostomy Tube Dislodgements } & Yes $(\mathbf{n}=7)$ \\
\cline { 2 - 4 } & No $(\mathbf{n}=\mathbf{2 7})$ & $65.14(21.78)$ & 0.299 \\
\hline Patient age, mean (SD) & $72.44(14.75)$ & $60.00(32.00)$ & 0.587 \\
Days observed, mean (SD) & $66.56(27.25)$ & $4(23.5)$ & 0.500 \\
Male gender, N (\%) & $13(76.5)$ & & \\
\hline
\end{tabular}


appropriately can lead to dreadful outcomes. Thus, a systematic and careful assessment of the underlying cause of each complication is essential to a successful enteral nutrition program.

Several reports explain particular strategies to minimizing or reducing the unintended dislodgement of gastrostomy tubes. Unfortunately, few approaches have been corroborated, other than changes in the shape or consistency of the internal bumper. A feasible approach would be the regular use of T-fastener/anchor systems to avoid disturbance of the gastrostomy tract if dislodgement did occur [5]. This approach has already been applied in laparoscopic approaches to gastrostomy formation and in the pediatric population in order to stabilize the recently developed gastrocutaneous fistula and permit appropriate maturation of the tract $[13,14]$.

Another means by which unintended dislodgement could be lessened is through intentional replacement with a gastrostomy tube of the type with a balloon [5]. This could occur only after complete maturation of the gastrocutaneous fistula tract (about 6 - 8 weeks) and would necessitate an additional outpatient clinic visit. It is plausible that a filled balloon would not pass as effortlessly through the gastrostomy tract and become dislodged. The great majority of initial gastrostomy tubes are inserted percutaneously and, as a result, do not have an existing balloon as the inner component.

To avoid devastating consequences, health care providers should be given appropriate instruction in managing gastrostomy tubes and their potential complications. It is also crucial that patient care decisions be made with full understanding of the patient's medical and surgical history, and are conveyed successfully to all members of the health care team. Furthermore, periodic review of practice may reveal opportunities for improvement. Both physician and nurse champions can facilitate change processes to improve practice and the delivery of care.

As demonstrated by our study, the implementation of a concise protocol to care for patients' gastrostomy tubes, which is attentively followed by the health care team, reduces unintended dislodgement. The next step after this pilot study is to implement this protocol across more units of the hospital, in a larger number of patients, and over a longer duration in order to achieve the maximum benefit for patient care.

\section{Acknowledgments}

The authors of this research study would like to express gratitude to Linda Deacon, statistical consultant, for her assistance with the data analysis, construction of the tables and insight during the course of this research.

\section{Conflict of Interest}

The authors of this research study declare that they have no competing interests. The authors declare that they have no affiliations with or involvement in any organization or entity with any financial interest or non-financial interest (such as personal or professional relationships, affiliations, knowledge or beliefs) in the subject matter discussed in this manuscript.

\section{Financial Support}

The authors of this research study declare that no financial support nor grant support has been received for the preparation of this manuscript.

\section{References}

1. Williams TA, Leslie GD, Leen T, Mills L, Dobb GJ. Reducing interruptions to continuous enteral nutrition in the intensive care unit: a comparative study. J Clin Nurs. 2013;22(19-20):2838-2848.

2. Slater R. Percutaneous endoscopic gastrostomy feeding: indications and management. Br J Nurs. 2009;18(17):1036-1043.

3. Kurien M, McAlindon ME, Westaby D, Sanders DS. Percutaneous endoscopic gastrostomy (PEG) feeding. BMJ. 2010;340:c2414.

4. O'Rear JM, Prahlow JA. Early percutaneous endoscopic gastrostomy tube dislodgment. Am J Nurs. 2015;115(6):26-31; quiz 32, 46.

5. Rosenberger LH, Newhook T, Schirmer B, Sawyer RG. Late accidental dislodgement of a percutaneous endoscopic gastrostomy tube: an underestimated burden on patients and the health care system. Surg Endosc. 2011;25(10):3307-3311.

6. Rosenberger LH, Guidry CA, Davis JP, Hranjec T, Johnston VK, Wages NA, Watson CM, et al. Reducing Accidental Dislodgement of the Percutaneous Endoscopic Gastrostomy: A Prospective Trial of the "SafetyBreak" Device. Surg Innov. 2016;23(1):62-69.

7. Schapiro GD, Edmundowicz SA. Complications of percutaneous endoscopic gastrostomy. Gastrointest Endosc Clin N Am. 1996;6(2):409-422.

8. Gavi S, Hensley J, Cervo F, Nicastri C, Fields S. Management of feeding tube complications in the long-term care resident. Annals of Long-Term Care: Clinical Care and Aging. 2008;16(4):28-32.

9. Santos PM, McDonald J. Percutaneous endoscopic gastrostomy: avoiding complications. Otolaryngol Head Neck Surg. 1999;120(2):195-199.

10. Rino Y, Tokunaga M, Morinaga S, Onodera S, Tomiyama I, Imada T, Takanashi Y. The buried bumper syndrome: an early complication of percutaneous endoscopic gastrostomy. Hepatogastroenterology. 2002;49(46):1183-1184.

11. Anagnostopoulos GK, Kostopoulos P, Arvanitidis DM. Buried bumper syndrome with a fatal outcome, presenting early as gastrointestinal bleeding after percutaneous endoscopic gastrostomy placement. J Postgrad Med. 2003;49(4):325-327.

12. Siegel TR, Douglass M. Perforation of an ileostomy by a retained percutaneous endoscopic gastrostomy (PEG) tube bumper. Surg Endosc. 2004;18(2):348.

13. Terry NE, Boswell WC, Carney DE, Beck A, Lowe L, 
Rittmeyer C. Percutaneous endoscopic gastrostomy with T-bar fixation in children and infants. Surg Endosc. 2008;22(1):167-170.
14. Duh QY, Way LW. Laparoscopic gastrostomy using T-fasteners as retractors and anchors. Surg Endosc. 1993;7(1):60-63. 